\title{
Introduction of robotic surgery for endometrial cancer is associated with lower bill sizes: The experience of a tertia- ry oncological centre in Singapore
}

\author{
Jeslyn Wong, Joseph Ng \\ Department of Obstetrics and Gynaecology, National University Hospital, Singapore, Singapore
}

Objective: In this exploratory study, we aim to present the effects of a shift in the standard of care from open to robotic surgery in endometrial cancer in the single largest series in Singapore, on total hospital bill size.

Methods: We retrospectively collected de-identified data from January 2008 to December 2017, consisting of all open and robotic surgeries performed for endometrial cancer in a tertiary oncology centre in Singapore. The data collected consisted of the number of open and robotic cases done per annum, and the average bill size incurred by patients for both routes of surgery. We analyzed the trend of the data over 10 years.

Results: There was a steady increase in the number of robotic hysterectomies performed over 10 years, from six robotic cases in 2008 (13.6\%), to 41 cases in 2017 (60.2\%). The number of open cases performed for endometrial cancers decreased from 38 cases (86.4\%) to 27 cases in 2017 (39.7\%). The total bill size decreased, from an average of SGD 15809.05 in 2012 to SGD 12891.00 in 2017. Conversely, the total bill size of open surgery rose from an average of SGD 13804.36 in 2012 to SGD 16243.43 in 2017.

Conclusion: This pilot data suggests that with the increase in uptake of robotic surgery for endometrial cancer, there was significant decrease in total bill size for patients who underwent robotic surgery.

Key Words: Robotic surgery; Endometrial cancer; Cost

\section{INTRODUCTION}

Endometrial cancer is the fourth most common cancer and the most common gynecological cancer in Singapore women [1]. The incidence of endometrial cancer has been steadily rising over the last three decades and it is likely to continue to do so due to demographic and socioeconomic factors such as rising rates of obesity and decreasing parity in Singapore [2,3]. Obesity and the fact that surgery is curative in most women with endometrial cancer has led to the gradual increase in the use of minimally invasive surgery (MIS) in the surgical management of endometrial cancer, with data to suggest that MIS should be the standard of care in endometrial cancer and especially in obese patients

- Received: March 5,2020 • Revised: May 20, 2020 • Accepted: July 21, 2020

- Correspondening author: Jeslyn Wong

Department of Obstetrics and Gynaecology, National University Hospital, NUHS Tower Block, Level 12, 1E Kent Ridge Road, Singapore 119228, Singapore E-mail: jeslyn_wong@nuhs.edu.sg

This is an Open Access article distributed under the terms of the Creative Commons Attribution Non-Commercial License (http://creativecommons.org/ licenses/by-nc/4.0) which permits unrestricted non-commercial use, distribution, and reproduction in any medium, provided the original work is properly cited. 
[4-6]. Robotic surgical platforms were first approved for gynecological surgery by the Food and Drug Administration in 2005. Robotic surgical technology has allowed more surgeons to offer minimally invasive gynecological surgery, shifting surgical loads away from open to MIS. The rapid adoption of robotic surgery in gynecology is multifactorial, but the main driver is the introduction of technology that bridges the skills gap between open surgery and traditional laparoscopy [7-9]. The performance characteristics of the robotic platform allows for a smoother transition from open surgical expertise to MIS and changed the demographics of the surgical management of endometrial cancer [5,9].

The majority of patients who receive surgical care in Singapore do so within the public healthcare system. The public healthcare system's payor mechanism is based primarily on a portion of each individual's private social security account set aside to cover healthcare costs, aptly named, Medisave. Employers pay into their employees' accounts in the program which is managed by the Singapore government through the Central Provident Fund (CPF). Medisave covers up to $80 \%$ of the consolidated hospital bill for each surgical care episode. Robotic and laparoscopic approaches in Singapore within the public health system are coded identically and so receive identical payor coverage. All public healthcare institutions that provide robotic surgical care have also adopted a "per case amortization" model where each case is charged a flat "facility fee" that is meant to repay the capital cost of the equipment and the cost of the maintenance contract. This fee is included in the total hospital bill.

To date, there has been no local data published on the effect of cost and hospital bill sizes on endometrial cancer care when the standard of care shifts directly from open to robotic surgery. Singapore data suggests that the immediate postoperative outcomes are better with robotic surgery compared to open and achievable by laparoscopy-naive surgeons [9]. The most directly relevant study that examined the impact of cost in a public healthcare system when the standard of care shifted from open surgery to robotic surgery for endometrial cancer was by Lau et al. [10]. The conclusion was that even accounting for amortization of the capital cost, robotic surgery was more cost effective than open surgery as a surgical care modality in endometrial cancer [10]. In this exploratory study, we present the effects of a shift in the standard of care from open to robotic surgery in endometrial cancer in the single largest series in Singapore and the effect of this shift on total hospital bill size.

\section{MATERIALS AND METHODS}

We retrospectively collected de-identified data from January 2008 to December 2017, including all open and robotic surgeries performed for apparent early-stage endometrial cancers in a tertiary oncology centre in Singapore, by identifying them using surgical logs and surgical codes. All apparent advanced endometrial cancers such as those with obvious extrauterine involvement were excluded. A total of 571 cases were analyzed: 319 open surgeries and 252 robotic. Data collected was exported to SPSS, version 25 (IBM Corp., Chicago, IL, USA), and consisted of the number of open and robotic cases done per annum, as well as the mean bill size incurred by the patients for both routes of surgery. Independent $t$-test analysis was done to determine if there are any statistical differences between the mean bill size for robotics and open surgery. For all $P<0.05$, $P$ was considered statistically significant. Exemption of Institutional Review Board review was obtained because it involved collection of existing data that was de-identified.

\section{RESULTS}

There was a steady overall increase in the number of robotic hysterectomies performed over the 10 years (Fig. 1).

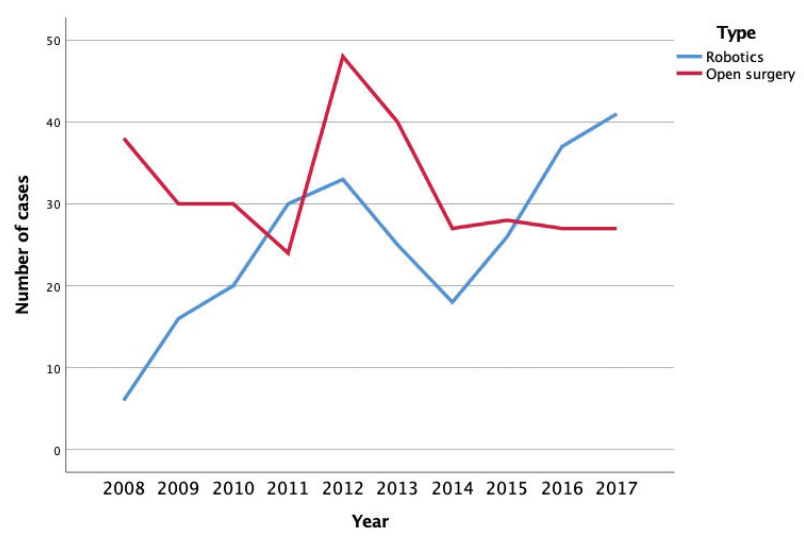

Fig. 1. Graph showing the trend in total annual number of open and robotic surgeries for endometrial cancer from January 2008 to December 2017. 
The number increased from six robotic cases in 2008 (13.6\%) to 41 cases in 2017 (60.2\%). On the other hand, the number of open cases performed for endometrial cancers decreased from 38 cases (86.4\%) to 27 cases in 2017 (39.7\%). There was a significant increase in proportion of robotic cases between 2012 and 2017 (19\%). From 2014 to 2017, open surgery cases remain within the range of 27 to 28 cases while robotics surgery cases increased from 18 to 41 cases, nearly double the robotic cases in 2014. Table 1 shows the number and proportions of open and robotic cases by year.

The mean total bill size by year is shown in Table 1 . The cost of robotics surgery dropped significantly from 2012 (mean, S\$15809.05; standard deviation [SD], S\$1982.96) to 2017 (mean, S\$12891.00; SD, S\$3190.38), with a mean difference of $S \$ 2918.05, t(68)=4.814, P<0.001$. This drop is not observed in open surgery. The cost of open surgery increased from 2012 (mean, S\$13804.36; SD, S\$6150.27) to 2017 (mean, S\$16243.43; SD, S\$9982.65), with no statistical difference between the years $t(73)=-1.311, P=0.194$. The trend in mean total bill size is depicted in Fig. 2.

\section{DISCUSSION}

There is no published data on the cost of robotic surgery for the most common gynecological cancer in Singapore, endometrial cancer. Local data and experience are particularly important as robotic surgery matures in Singapore and there is increasing interest in the issue of the value that each intervention adds to public healthcare. The cost to patients of robotic surgery has been one of the main concerns for healthcare institutions and systems. One area of immediate concern is the significant initial investment involved in the acquisition of the robotic surgical platform, and the maintenance contract over the service lifetime of the system. The cost of this initial investment and the service contract are often amortized against each surgical case over

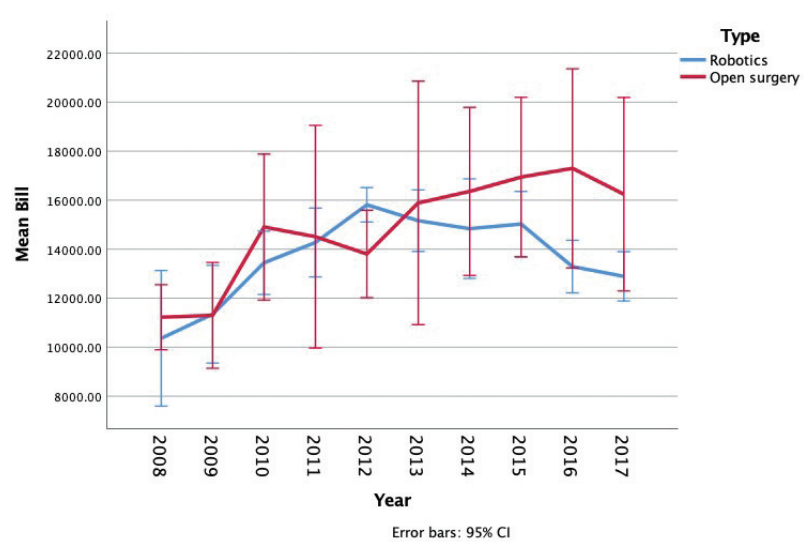

Fig. 2. Graph showing the trend in annual mean total bill size (SGD) for open and robotic surgeries for endometrial cancer from January 2008 to December 2017.

Table 1. Number of open and robotic surgeries for endometrial cancer and the annual mean total bill size from 2008 to 2017

\begin{tabular}{lccccc}
\hline \multirow{2}{*}{ Year } & \multicolumn{2}{c}{ Open } & & \multicolumn{2}{c}{ Robotic } \\
\cline { 2 - 3 } \cline { 5 - 5 } & No. of cases per annum & Mean total bill size (SGD) & No. of cases per annum & Mean total bill size (SGD) \\
\hline 2008 & $38(86.0)$ & $11,223.73$ & $6(14.0)$ & $10,360.74$ \\
2009 & $30(65.0)$ & $11,298.05$ & $16(35.0)$ & $11,347.49$ \\
2010 & $30(60.0)$ & $14,902.57$ & $20(40.0)$ & $13,444.39$ \\
2011 & $24(44.0)$ & $14,509.34$ & $30(56.0)$ & $14,273.55$ \\
2013 & $48(59.0)$ & $13,804.36$ & $33(41.0)^{\mathrm{a})}$ & $15,809.05^{\mathrm{b})}$ \\
2014 & $40(62.0)$ & $15,887.56$ & $25(38.0)$ & $15,161.58$ \\
2015 & $27(60.0)$ & $16,357.85$ & $18(40.0)$ & $14,838.06$ \\
2016 & $28(52.0)$ & $16,940.77$ & $26(48.0)$ & $15,026.82$ \\
2017 & $27(42.0)$ & $17,299.80$ & $37(58.0)$ & $13,289.19$ \\
Total & $27(40.0)$ & $16,243.43$ & $41(60.0)^{\mathrm{a})}$ & $12,891.00^{\mathrm{b})}$ \\
\hline
\end{tabular}

Values are presented as number (\%).

a) Statistically significant increase in proportion of robotic cases between 2012 and 2017; b) Statistically significant decrease in mean total bill size between 2012 and 2017. 
the service lifetime of the system as a "facility fee". This "upfront fee" in addition to any consumables, instruments, and logistics that are involved in each surgical case usually result in a significantly higher hospital bill size, and has not changed over the duration of study. This form of cost recovery is commonly used in Singapore and throughout Asia. This should be taken into account when considering the actual cost of robotic surgery against those of other minimally invasive surgical modalities. Other factors that need to considered when addressing the issue of cost are overall surgical load, proportion of the total surgical load that is performed open, potential savings by converting the open load to MIS, and what modality can most efficiently deliver this conversion from open to MIS. Lau et al. [10] showed that in a public healthcare payor system with a significant endometrial cancer load, converting from open to robotic represented savings mainly because of shorter hospital stays, and fewer complicated readmissions, which resulted in lower bill sizes as compared to their historical cohort, in which majority of their patients had open surgery. Our pilot data suggests that findings by Lau et al. [10] may be applicable to programs in Singapore as well, and that the total cost of providing surgical care for endometrial cancer is lower with robotic surgery as compared to open surgery.

When considering the larger picture of societal costs, Barnett et al. [11] demonstrated that robotic surgery is less "costly" than open surgery. The depreciation cost of the robot system did not affect the patient's bill size during the period of study.

With the introduction of robotic surgery in our centre in 2008, the groundwork was set for a pilot program to offer day-surgery hysterectomy. Few patients were discharged on the same day after robotic surgery then. However, since 2016, the default pathway for patients who have had a hysterectomy for endometrial cancer is for discharge on the same day of surgery. This has been shown to be a safe, effective, and feasible care model for our patients, as an internal audit on day surgery cases performed from March 2016 to December 2016 led to no operative complications, and no re-admissions ( $n=14)$.

In this study, we demonstrated the effect of increased surgical efficiency and the economies of scale on the patient's total hospital bill size. As surgeons and surgical teams leverage robotic surgical technology to allow for greater daily throughput in the period described from 2012 to 2017, the fixed cost of providing surgical care was increasingly offset by increased revenue, shorter hospital stays for a larger proportion of patients receiving surgical care, and a lower overall usage of inpatient resources.

Ultimately, the goal of adopting robotic surgery is to reduce the proportion of open surgeries, and not to convert laparoscopic procedures into robotic procedures. While conventional laparoscopy is apparently associated with lower costs as compared to both open and robotic surgery for endometrial cancer [11] the more important question remains as to which modality will "move the needle" towards MIS more efficiently. In gynecological cancer, the introduction of robotic surgery "moved the needle" from open to MIS in much more significant way than when laparoscopy was introduced. Moore's Law is often summarized as the doubling of computing power halves the cost of technology, this together with the ability of robotic surgical platforms to bridge the gap between open surgery and traditional laparoscopy translates into an increasingly more efficient model to equip more surgeons to deliver minimally invasive surgical care.

Since the goal of this study is to only analyze and consider the total bill size of the patient, the limitation is the lack of breakdown of the bill: hospital stay, cost incurred for operating room and equipment, and post-operative complications. Longitudinal data including re-admission data and costs is also unavailable. Future research including such detail and health economics studies can shed more light on the overall cost difference between the two routes of surgery. Additionally, there is no comparison with laparoscopic route of surgery for endometrial cancer. This is not within the scope of this study as the aim is to demonstrate how patients' bill sizes change in an institution which lacks laparoscopically trained gynae-oncologist, but are still providing patients with minimally-invasive cancer surgery through the bridging from open to robotic surgeries.

This pilot data suggests that with the increase in uptake of robotic surgery for endometrial cancer, there was significant decrease in total bill size for patients who underwent robotic surgery. Increase use of the robotic surgical platform, improved efficiency and shorter hospital stay with robotic surgery will likely continue to direct bill sizes downward. 


\section{Robot surgery in uterine cancer and cost $\mid$ Wong J, et al.}

\section{Conflict of interest}

No potential conflict of interest relevant to this article was reported.

\section{Acknowledgments}

We would like to thank Mandi for assisting us in statistical analysis, and Division of Gyne-Oncology, Department of Obstetrics and Gynecology, National University Hospital.

\section{References}

1. National Registry of Diseases Office (NRDO). Singapore Cancer Registry Annual Registry Report 2015 [Internet]. Singapore: NRDO; c2017 [cited 2020 Apr 15]. Available from: https://www.nrdo.gov.sg/docs/librariesprovider3/Publications-Cancer/cancer-registry-annual-report-2015_web. pdf?sfvrsn=10.

2. Executive Summary on National Population Health Survey 2016/17 [Internet]. Singapore: Ministry of Health; c2018 [cited 2020 Apr 15]. Available from: https://www.moh.gov.sg/docs/ librariesprovider 5/resources-statistics/reports/executivesummary-nphs-2016_17.pdf.

3. Department of Statistics Singapore. Births and Fertility 2019 [Internet]. Singapore: Department of Statistics Singapore; c2020 [cited 2020 Apr 15]. Available from: https://www. singstat.gov.sg/find-data/search-by-theme/population/birthsand-fertility/latest-data.

4. Janda M, Gebski V, Davies LC, Forder P, Brand A, Hogg R, et al.
Effect of total laparoscopic hysterectomy vs total abdominal hysterectomy on disease-free survival among women with stage I endometrial cancer: a randomized clinical trial. JAMA 2017;317:1224-33.

5. Leitao MM, Narain WR, Boccamazzo D, Sioulas V, Cassella D, Ducie JA, et al. Impact of robotic platforms on surgical approach and costs in the management of morbidly obese patients with newly diagnosed uterine cancer. Ann Surg Oncol 2016;23:2192-8.

6. Suidan RS, He W, Sun CC, Zhao H, Fleming ND, Ramirez PT, et al. Impact of body mass index and operative approach on surgical morbidity and costs in women with endometrial carcinoma and hyperplasia. Gynecol Oncol 2017;145:55-60.

7. Chen SH, Li ZA, Huang R, Xue HQ. Robot-assisted versus conventional laparoscopic surgery for endometrial cancer staging: a meta-analysis. Taiwan J Obstet Gynecol 2016;55:488-94.

8. Coronado PJ, Herraiz MA, Magrina JF, Fasero M, Vidart JA. Comparison of perioperative outcomes and cost of roboticassisted laparoscopy, laparoscopy and laparotomy for endometrial cancer. Eur J Obstet Gynecol Reprod Biol 2012;165:28994.

9. Mok ZW, Yong EL, Low JJ, Ng JS. Clinical outcomes in endometrial cancer care when the standard of care shifts from open surgery to robotics. Int J Gynecol Cancer 2012;22:819-25.

10. Lau S, Vaknin Z, Ramana-Kumar AV, Halliday D, Franco EL, Gotlieb WH. Outcomes and cost comparisons after introducing a robotics program for endometrial cancer surgery. Obstet Gynecol 2012;119:717-24.

11. Barnett JC, Judd JP, Wu JM, Scales CD Jr, Myers ER, Havrilesky LJ. Cost comparison among robotic, laparoscopic, and open hysterectomy for endometrial cancer. Obstet Gynecol 2010;116:685-93. 